\title{
Invisibilización de la mujer ecuatoriana en la sociedad actual
}

\author{
Carlos Calderón-Guevara \\ ccalderon@uce.edu.ec \\ https://orcid.org/0000-0002-7408-8733
}

Melany Daniela Chávez Buri

mdchavez@uce.edu.ec

\section{RESUMEN}

El presente artículo tiene como objetivo analizar la invisibilización de la mujer ecuatoriana en la sociedad actual. En el aparatado teórico se especifica el contexto en el cual se desarrolla la población femenina en el Ecuador. El estudio es cualitativo de carácter descriptivo, documental-bibliográfico. En la recolección de información se utilizó la técnica del fichaje y el instrumento las fichas de trabajo. En los resultados se afirma que el sistema patriarcal/capitalista causa la invisibilización femenina y por ende es una problemática que aún persiste en la sociedad; lo que hace que sectores importantes de mujeres asuman una posición contrahegemónica de cuestionamiento constante. Entre las principales conclusiones se establece que el proceso de invisibilización de la mujer nace del androcentrismo, se fortalece con la existencia del patriarcado que, como parte del sistema vigente, originan la vulneración permanente de los derechos de la mujer. Finalmente, se destaca el papel protagónico que juegan hoy en día las mujeres ecuatorianas para la construcción de una sociedad más justa.

Palabras clave: invisibilización; patriarcado; machismo; misoginia; capitalismo. 


\title{
Invisibility of ecuadorian women in today's society
}

\begin{abstract}
This article aims to analyze the invisibility of Ecuadorian women in today's society. In the theoretical section, the context in which the female population in Ecuador develops is specified. The study is qualitative descriptive, documentary bibliographical. In the collection of information, the recording technique and the worksheet instrument were used. In the results it is affirmed that the patriarchal/capitalist system causes the invisibility of women and therefore it is a problem that still persists in society; what makes important sectors of women assume a counter-hegemonic position of constant questioning. Among the main conclusions, it is established that the process of making women invisible is born of androcentrism, it is strengthened by the existence of patriarchy that, as part of the current system, causes the permanent violation of women's rights. Finally, the leading role those Ecuadorian women play today for the construction of a more just society is highlighted.
\end{abstract}

Keywords: invisibility; patriarchy; sexism; misogyny; capitalism

Artículo recibido: 15 enero 2022 Aceptado para publicación: 08 febrero 2022 Correspondencia: ccalderon@uce.edu.ec Conflictos de Interés: Ninguna que declarar 
"No seré una mujer libre mientras siga habiendo mujeres sometidas, incluso cuando sus cadenas sean muy diferentes a las mías."

Audre Lorde

\section{INTRODUCCIÓN}

La invisibilización es parte de una problemática que afecta a distintos grupos no hegemónicos, en el caso específico de las mujeres su invisibilización es la consecuencia de un sistema androcentrista, patriarcal y capitalista. El reconocimiento que se le da a la mujer a nivel de sociedad es casi nulo en los campos económicos, sociales, políticos, académicos, tecnológicos, deportivos, etc. En las actividades que se les toma en cuenta son en los quehaceres domésticos y tareas de cuidado, alegando que ellas las realizan de manera innata solo por ser mujeres.

Se han dado diversas acciones que pretenden reducir la problemática de género e invisibilización femenina, entre las más comunes están la paridad numérica su integración mínima, sin embargo, el número de mujeres que integren un espacio no tienen relación directa con la validez que se le da a sus opiniones o necesidades.

Al ser parte de una región con distintas problemáticas sociales, Ecuador también refleja la invisibilización de la mujer como un problema que violenta a la población femenina. Cuando se analiza la situación es necesario tomar en cuenta un punto trascendental, las mujeres ecuatorianas son diversas en todos los sentidos ya sea por su origen étnico, clase, género u otras. Las particularidades de cada mujer influyen mucho al hablar de su posición ante el reconocimiento, una mujer afroecuatoriana no está en la misma condición de privilegio que una mujer blanca. A pesar de sonar contradictorio, existen sectores de mujeres que se encuentran más invisibles e ignoradas que otras, a aquella situación se la puede denominar como otras de la otredad.

Este trabajo pretende dar respuestas a las siguientes preguntas: ¿Por qué se invisibiliza a la mujer ecuatoriana en nuestra sociedad?, ¿Cuáles son las consecuencias de la invisibilización de la mujer? y, ¿Qué papel ha tenido la mujer dentro de la sociedad?

Con relación a la estructura y contenido del artículo, en el primer apartado se encuentran las ideas iniciales, síntesis de la investigación y las bases teóricas. En el segundo apartado se realiza la descripción de la metodología investigativa. En el tercer apartado se presenta 
el análisis y discusión de resultados. Por último, en el cuarto apartado se exponen las conclusiones del presente estudio.

\subsection{Conceptos relacionados}

\subsubsection{Invisibilización de la mujer-Androcentrismo}

En el campo social la invisibilización es una palabra que se utiliza para hacer referencia a un problema social de no reconocimiento de un grupo determinado, esto conlleva a que dicho grupo sufra distintas consecuencias que lo convierte en vulnerable. En palabras de (Hernández, 2020) "Son muchos los teóricos que han recurrido a ella como una herramienta para la comprensión y explicación de la realidad social” (p. 102). Agregando la definición de (Honnet, 2011) "es el resultado de una deformación de aquella capacidad humana de percepción que está vinculada con el reconocimiento" (p. 181).

Tomando en cuenta la opinión de los dos autores, la palabra invisibilización es un término que busca enmarcar una problemática social, cuya característica es que la sociedad no percibe a un grupo como parte de esta y a la vez ignoran su existencia y todo lo que esta conlleva, es decir, condiciones, necesidades y exigencias.

Una vez entendida la problemática, es necesario explicar su origen, pues la mujer forma parte de un grupo que ha sido excluido históricamente. Este fenómeno nace del androcentrismo, el hecho de poner al hombre como representante de la humanidad, el punto máximo de la evolución y como un estándar irrevocable ha dado paso a que la mujer sea ignorada y no reconocida. A pesar de que ambos son parte de la especie humana, el hombre prevalece en la cúspide de la jerarquía social.

\subsubsection{Patriarcado}

A lo largo de la historia, las sociedades se han desarrollado con base a distintas formas de gobierno y organización social, una de las que más destaca es el patriarcado. El origen del nombre nace en Grecia y hace referencia al padre, dado que él era quien debía estar a cargo de todas las cuestiones familiares que se relacionen al poder y en su defecto, si este se encontraba ausente, el control lo tomaba el varón con más edad de la casa. En la actualidad este sistema social de poder se encuentra vigente, de su seno surgen actitudes machistas y misóginas que afectan directamente a las mujeres, el menosprecio y repudio a todo lo que se relacione con ellas, es un claro ejemplo de cómo los valores patriarcales se anteponen a su bienestar. 
El patriarcado es palpable en la realidad de las mujeres y en las relaciones de poder que se establecen entre los hombres. Cuando los varones se relacionan con sus pares se crean vínculos de apoyo que les facilita la subordinación de mujeres, ya sea de manera consciente o inconsciente. Aunque este sector de la población posea diferencias de etnia, clase, etarias u otras, las alianzas constituidas persisten en la mayoría de las ocasiones, los argumentos más comunes para menospreciar e invalidar a una mujer son principalmente económicos, étnicos, biológicos y religiosos.

\subsubsection{Mecanismos de invisibilización}

El grupo de poder que lidera el patriarcado utiliza diversos mecanismos para ejercer la invisibilización, desde los sesgos de género hasta el ideario de la elección familiar libre; en situaciones comunes y las maneras de silenciar a las mujeres, pueden expresarse así en la vida cotidiana:

- Mansplaining, Término usado para hacer referencia a la actitud de los hombres cuando explican algo a una mujer desde una postura paternalista y condescendiente, como si no tuviera los conocimientos o inteligencia suficiente para entender algo. (Vaccaro, 2021, p. 199).

- Manterrupting: Interrumpir intencionalmente por parte de un hombre a una mujer hasta que ella decida guardar silencio, situación que normalmente no sucede entre hombres. (Leal, 2019, p. 355).

- Gaslighting Hacer sentir a una persona que se está volviendo "loca" o perdiendo la cordura, invalidando sus ideas y sentimientos. (Galán \& Figueroa, 2017, pp. 57-58).

Estos mecanismos cotidianos son parte de las acciones que se generan a diario para que los hombres consigan silenciar a las mujeres y, por consecuencia, esto se replica en mayor magnitud a nivel social e institucional donde se encuentran normalizadas estas formas de pensar, sentir y actuar. La naturalización del menosprecio hacia la mujer es causal de su invisibilización en todos los estratos y campos de la sociedad.

\subsubsection{Patriarcado y capitalismo}

El patriarcado y el capitalismo están unidos por el mismo cordón umbilical, se desenvuelven y fortalecen simultáneamente, a pesar de que el primero existe mucho antes de la aparición del capitalismo; el día de hoy ambos juegan un papel preponderante en la realidad de las mujeres. Por un lado, el patriarcado predica la superioridad del hombre y 
por otro, el capitalismo, ha desvalorizado socialmente el trabajo doméstico y de cuidado, destinado principalmente a ellas.

Los capitalistas y sus detractores no conciben al trabajo doméstico realizado por mujeres como una actividad que genere réditos económicos, por ende, se lo ignora y no se le da la atención necesaria, es más este no es considerado como trabajo o actividad laboral. Adicional a esto, el capitalismo promueve la explotación de las mujeres, de manera principal en el campo de lo sexual, sexualización y mercantilización de los cuerpos para obtener más ganancias a través de la pornografía, la prostitución y el proxenetismo.

Con la finalidad de comprender la relación entre patriarcado y capitalismo, si es un sistema unificado o dos sistemas independientes, (Frega, 2019, pp. 23-26), menciona a varios autores/as que exponen sus principales argumentos de las dos teorías:

\section{Teoría de los dos sistemas}

- Heidi Hartmann: Las jerarquías establecidas por el orden patriarcal sientan las bases materiales que ordenan la división sexual del trabajo y estas se entrelazan con los mecanismos de explotación capitalista.

- Christine Delphy: Existencia de un modo de producción doméstico propio del sistema patriarcal. Las relaciones entre las clases antagónicas se trasladan directamente a las relaciones sexuales de explotación y opresión de los hombres hacia las mujeres.

\section{Teoría unitaria}

- Iris Young: Se incorpora al análisis de la opresión capitalista a la categoría de género con relación al trabajo productivo y reproductivo.

- Cinzia Arruzza (2016) y Tithi Batthacharya (2015): Relaciones patriarcales de dominación son constitutivas de las estructuras e instituciones sociales capitalistas, conformando así un sistema unificado de explotación y dominación.

Ahora bien, el capitalismo patriarcal o el patriarcado del capital son sistemas que de una $\mathrm{u}$ otra forma generan estructuras y relaciones de poder para conseguir la vulneración de los derechos de la mujer, sin importar que se los considere un sistema unitario o dos sistemas separados que se interrelacionan. Es verdad que un hombre proletario y un hombre burgués no poseen los mismos privilegios de clase, a pesar de que, el primero sufra la explotación del segundo, el hombre proletario puede continuar con la opresión de la mujer dentro de su realidad. 
Amoros (1992), afirma que "De no haber existido el patriarcado la clase obrera unificada podría haberse enfrentado al capitalismo, pero las relaciones sociales patriarcales dividieron a la clase obrera, permitiendo que una parte (los hombres) fuera comprada a expensas de la otra (las mujeres)” (p. 43). Desde una visión anticapitalista el autor cuestiona el carácter del patriarcado como causante de la situación actual que vive la mujer en general e implícitamente cuestiona al capitalismo, pues son sistemas de opresión que afectan a las mujeres, no sería de mucha ayuda que se acabe con la explotación de las mujeres a nivel de sistema económico, si dentro de sus hogares siguen siendo relegadas al cumplimiento de tareas domésticas.

\subsubsection{Invisibilización de mujeres ecuatorianas}

Ecuador se enmarca y desarrolla dentro del capitalismo y el patriarcado, por ende, las mujeres ecuatorianas sufren la invisibilización generada por estos sistemas. Según las cifras del INEC (2010), el 50,44\% de la población está conformada por mujeres, es decir la población femenina es mayor que la masculina, pero a pesar de existir superioridad numérica las mujeres no tienen los mismos beneficios que los hombres a nivel general. Es necesario mencionar que al hablar de generalidad se hace referencia a la invisibilización que sufren las mujeres sin tomar en cuenta las categorías de etnia, clase, orientación sexual o diversidad sexo genérica.

En este país se pueden reconocer actitudes de discriminación de manera cotidiana. Coello (2016) asevera que "A pesar de que la cultura ecuatoriana es machista y racista, la mayoría de la sociedad no reconoce serlo, aunque en la vida diaria se demuestre lo contrario" (p. 108). De acuerdo con Coello, la mayoría de la población ecuatoriana niega la existencia de las problemáticas de exclusión, pero en la realidad se evidencian varias conductas que prueban su plena vigencia en las relaciones sociales y humanas. El ignorar por completo la existencia de una problemática da paso a que se generen nuevas barreras para las mujeres.

Todas las actividades de la sociedad, con excepción las tareas del hogar o de cuidado, continúa manejadas por los hombres, indicador que permite afirmar que existe discriminación, desigualdad e inequidad, se vulneran derechos de las mujeres en actividades vinculadas con la política, salud, economía, educación o cualquier sector productivo. El creer que integrar a las mujeres a los distintos espacios de participación elimina la problemática de reconocimiento y valoración es una idea errada, no solo es 
necesario que formen parte de estos espacios, sino que es vital que sean escuchadas y consideradas como iguales, con derechos y responsabilidades.

Un problema que afecta al reconocimiento femenino en cualquier ámbito es el uso del masculino genérico, el sexismo dentro del lenguaje y los discursos con misoginia y machismo implícito, es por este motivo que el Consejo de Regulación y Desarrollo de la Información y Comunicación, CORDICOM (2017) asevera que los "discursos diferenciales hacia las mujeres, (...) constituyen representaciones negativas $\mathrm{y}$ estereotipadas de la población femenina, elementos que refuerzan la ideología patriarcal y configuran un discurso de carácter sexista, que históricamente ha valorado al hombre" (p. 305). La forma en la cual el ser humano se comunica y el mensaje que transmite genera consecuencias en la realidad de los diversos sectores de la sociedad, específicamente para las mujeres, se piensa que el término todos es utilizado para englobar tanto a hombres como a mujeres, a pesar de ello, lo que no se nombra no es percibido por la sociedad y genera problemáticas de omisión, pues se habla explícitamente de hombres y se sobreentiende que ellas forman parte de ese grupo.

Por otro lado, dentro del campo de la política ecuatoriana las mujeres aún no comparten las mismas condiciones que los varones para lograr sobresalir en el mismo. Es verdad que existen mujeres dentro de los distintos cargos políticos, pero esto no garantiza que se les de la misma validez y reconocimiento que reciben los hombres. Varias organizaciones como ONU MUJERES \& CNE (2019) concluyen que:

(...) más allá de que en algunos partidos pueda haber una mayor presencia numérica de mujeres y ellas ocupen cargos de dirección, siguen manteniendo una situación de subordinación respecto de los hombres. Aunque las mujeres afiliadas/militantes participan y opinan al interior de los partidos, siempre se tiende a dar la razón a los hombres. (p. 29)

De estas afirmaciones se deduce lo siguiente, el número de mujeres que integren un partido u organización política no son garantía de que sean tomadas en cuenta, en otras palabras, las opiniones, ideas y decisiones de los varones tienen mayor validez al hacer política en comparación a las que puedan emitir las mujeres. Cuando se habla de activismo político y militancia existe una superioridad masculina, aún en este campo las mujeres son relegadas 
a tareas de logística porque se cree que son actividades que pueden realizar por su naturaleza, ignorando sus potencialidades y capacidades de liderazgo y de gestión.

El mundo de la ciencia y tecnología no ha presentado condiciones distintas para el desenvolvimiento de las mujeres, existe androcentrismo, machismo y misoginia. En este sector se propicia el raciocinio de los hombres, pues a las mujeres se las considera sentimentales y por este motivo no son personas indicadas para generar ciencia. (Pessina, 2019) analiza la situación de las mujeres en la ciencia y tecnología ecuatoriana, plantea que efectivamente existe una igualdad legal; las leyes, normas y reglamentos describen a hombres y mujeres como seres iguales ante el Estado, sin embargo a nivel social esto no sucede, los roles y estereotipos de género afectan la participación científica femenina, además de ser objeto de una segregación (pp. 53-54).

Los deportes también son parte de la invisibilización, en este campo, los prejuicios de la sociedad lo han dividido en aquellos que son para hombres o mujeres; los primeros realizarán actividades deportivas de agilidad, velocidad y sobre todo de fuerza, por el contrario, las mujeres serán parte de deportes que socialmente son considerados femeninos. Aún con esta clasificación no oficial del deporte las mujeres no logran ser protagonistas, (Ordóñez, 2011) determina que existe poca población femenina practicante de un deporte profesional en áreas urbanas misma que es aún peor en la ruralidad, los factores que afecta al deporte de mujeres son: las jornadas laborales, el trabajo doméstico, el monopolio del futbol masculino y las constantes violencias hacia su aspecto físico o manera de vestir, sin mencionar la falta de incentivos de los gobiernos. (pp. 112-113)

En este sentido las mujeres no son un grupo protagónico en el campo de los deportes, aquellas que son parte de este campo pasan desapercibidas por los medios de comunicación, adicional a eso, se da más énfasis a su validación como objetos sexualizados que deben ser aceptados por los hombres. Finalmente, los gobiernos no han implementado políticas que fomenten e incentiven la integración e inclusión de las mujeres en el deporte tomando en cuenta todos los factores que influyen en realidad.

De acuerdo con los datos del Instituto Nacional de Estadística y Censos (INEC, 2020) las mujeres representan el $77,4 \%$ de trabajo no remunerado en la zona urbana y $76,9 \%$ en la zona rural, en comparación a un 22,85\% de hombres. Las mujeres son quienes sostienen el hogar, con la incorporación de las mujeres al campo laboral sus jornadas de trabajo se han intensificado, hasta dos o tres veces más. El trabajo laboral, el trabajo doméstico y de 
cuidado que deben realizar representa un gran aporte para la familia y la sociedad, a pesar de ello no es reconocido. (Radcliffe, 2014) afirma, el trabajo doméstico y no remunerado es invisibilizado por las reglas patriarcales sobre el género, por este motivo los trabajadores no logran entender completamente las limitaciones que atraviesan las mujeres. (p. 22)

Como sostiene el autor, las mujeres se desarrollan en condiciones distintas a los hombres, el trabajo doméstico se encuentra naturalizado como una actividad innata de ellas, por lo cual no es considerado relevante. En la mayoría de los casos, los trabajadores finalizan su jornada de trabajo al llegar a su casa, situación que no se replica a las mujeres. Ellas se levantan antes para cocinar u ordenar la casa, van a trabajar, regresan, se hacen cargo del aseo, cuidado de los hijos/as e incluso cumplen funciones de docentes al realizar tareas escolares.

Uno de los puntos más graves de la invisibilización de las mujeres se refleja en el no reconocimiento de la violencia en cualquiera de sus formas. Según datos proporcionados por el Instituto Nacional de Estadísticas y Censos (INEC, 2019), el 64,9\% de la población femenina ha vivido algún tipo de violencia relacionada al género a lo largo de su vida a nivel nacional, sin embargo, la mayoría prefiere no denunciarla ante las instituciones pertinentes.

Los resultados obtenidos en la investigación realizada por la Fiscalía General del Estado y la Escuela Politécnica Nacional (2020) indican que es mínimo el porcentaje de denuncias por violencia de género; en promedio, el $95 \%$ no denuncia la violencia física, el $84 \%$ la violencia sexual y, el $85 \%$ la violencia psicológica. Entre las razones más comunes para que decidan no continuar o iniciar una denuncia, están los prejuicios de policías, jueces y abogados, además de la revictimización y cuestionamiento de sus acciones. La expresión culminante de la violencia es el femicidio, hasta 2020 existía un promedio de 6 delitos por mes; a pesar de ello, colectivos y organizaciones afirman que el número es más alto que los datos oficiales.

El no tomar en cuenta las problemáticas de las mujeres provoca que sus condiciones de violencia y vulnerabilidad se agraven, la naturalización del uso genérico del masculino, la revictimización, poner en tela de juicio el accionar de las víctimas y la impunidad en todos los casos son condicionantes para ellas. Es pertinente mencionar que esto disminuye las probabilidades de que las víctimas compartan sus experiencias y a su vez la posibilidad de crear proyectos preventivos y de ayuda en situaciones de violencia de género. 
En Ecuador existen una amplia diversidad de mujeres, las categorías de etnia, clase, género, orientación sexual y diversidad sexo genérica juegan un papel importante al momento de analizar las problemáticas que viven a diario. No se pueden comparar las violencias que afectan a las mujeres afroecuatorianas, indígenas, lesbianas y/o trans con las que sufren las mujeres hegemónicas, blanco-mestizas, heterosexuales y cisgénero. El racismo se liga con el patriarcado y el capitalismo para discriminar a mujeres afroecuatorianas e indígenas. Adicionalmente, el patriarcado que impone la heteronormatividad y el cisgenerismo crea barreras para el desarrollo de lesbianas y mujeres trans a nivel nacional.

\subsubsection{Aporte de la mujer}

Es importante resaltar que, a lo largo de la historia ecuatoriana, el aporte de la mujer se ha dado en diversos campos, y su lucha ha impulsado el logro de derechos, como el derecho al voto y la participación política; además de procesos emancipadores por la equidad, sin olvidar la resistencia indígena, afro, entre otros; en las cuales las mujeres han representado un papel fundamental.

En la actualidad, en el campo económico, las mujeres también tienen un rol crucial para el desarrollo del país. (Acosta, 2019) afirma que el trabajo femenino es muy representativo para el crecimiento económico de la nación ecuatoriana en las actividades de comercio, la agricultura, la ganadería y la manufactura. (p. 53) Adicionalmente a lo que menciona Acosta, se debe hablar sobre el aporte del trabajo doméstico, los hombres no podrían desarrollarse de la manera en que lo hacen si las mujeres no asumieran todo el trabajo doméstico y de cuidado, siendo una contribución objetiva, invisibilizada y desvalorizada. La participación de las mujeres en la esfera política y social es el resultado de su lucha y organización colectiva. Ortiz (2012) afirma que "la tenacidad en la lucha de las mujeres (es) la que les ha permitido ir ganado espacios en lo social y concretamente en lo político y hacer que se las visibilice y valore su aporte" (p. 85). Por tanto, los espacios ganados por las mujeres en algunas esferas de la vida económica, social, política y cultural, y donde participan activamente, son el fruto de la constancia, hacer escuchar su voz y lucha permanente que le han dado al sistema; es decir, la participación de la mujer ha obligado al sistema y sus representantes que se la visibilice, aunque falte mucho por hacer y conseguir. Con una mirada retrospectiva, es necesario reconocer el significado que han tenido en la historia nacional destacadas mujeres en la lucha social y política por sus derechos y reivindicaciones, mujeres como: Manuela Saénz, Manuela Espejo, Marieta de Veintimilla, 
Rosa Cabeza de Vaca, Matilde Hidalgo de Prócer, Zoila Ugarte de Landívar, Nela Martínez, María Zúñiga, Transito Amaguaña, Dolores Cacuango, Sonia España Quiñónez, Rosa Paredes Jumbo y una infinidad de mujeres, han luchado desde sus realidades y fueron quienes sentaron las bases del proceso reivindicativo pero que la historia las ha invisibilizado.

Es importante destacar la creación de redes de apoyo y organización para sobrellevar las dificultades que se puedan presentar, tanto la Red Ecuatoriana de Mujeres Científicas (REMCI), Coordinadora Nacional de Mujeres Negras (CONAMUNE), Mujeres CONAIE, Mujeres por el Cambio, Las Comadres, La Kasa de acogida trans y otros colectivos que organizan por la necesidad de establecer espacios seguros que les permitan expresarse y representen un soporte para ellas.

\section{ESTRATEGIAS METODOLÓGICAS}

En este apartado se describe la metodología utilizada en la investigación:

Enfoque: Se utiliza la metodología cualitativa orientada al análisis crítico de los datos obtenidos de la información documental, apoyándose en el método inductivo.

Nivel de profundidad: El nivel de profundidad es descriptivo, lo que permite caracterizar la problemática identificada.

Tipo de investigación: La investigación es documental, fundamentada en la recolección, revisión y análisis de la información obtenida en textos físicos y digitales.

Técnicas e instrumentos: Para la recolección de información se empleó la técnica del fichaje y el instrumento, la ficha de trabajo, lo que permitió la recolección, sistematización y organización de la información documental.

Técnica de procesamiento de información: Se emplea la técnica de análisis de contenido cualitativo. La técnica consiste en realizar un cotejo y comparación de lo que afirman las y los diferentes autores, adicional a eso, este análisis se complementa con las interpretaciones de la investigadora.

\section{RESULTADOS Y DISCUSIÓN}

El estudio se enfoca en dar respuesta a las preguntas: ¿Por qué se invisibiliza a la mujer ecuatoriana en nuestra sociedad?, ¿Cuáles son las consecuencias de la invisibilización de la mujer? y, ¿Qué papel ha tenido la mujer dentro de la sociedad?

Con relación a la interrogante ¿Por qué se invisibiliza a la mujer ecuatoriana en nuestra sociedad?, la invisibilización de la mujer ecuatoriana es el producto de diversos sistemas 
de opresión. El más preponderante es el patriarcado, el cual se articula con el sistema capitalista, siendo estos los causantes de crear las condiciones de vulnerabilidad e invisibilización de las mujeres. Amoros (1992) establece que, si no hubiera existido el capitalismo la clase obrera estuviera unificada, pero al no ser así se generan relaciones de verticalidad dentro de una misma clase social poniendo en desventaja a las mujeres (p.43). De acuerdo con lo recopilado por Frega (2019), la teoría de los dos sistemas de Hartmann y Delphy, determinan al patriarcado y al capitalismo como dos sistemas independientes pero relacionados entre sí; o el sistema unitario que sostiene Arruzza Batthacharya, y Young que establece la existencia de un sistema único, es decir, la conjunción entre patriarcado y capitalismo da paso a la invisibilización de la mujer. (pp. 23 - 26)

Además, las mujeres afroecuatorianas e indígenas en el país deben afrentar la problemática del racismo y etnocentrismo. Coello (2016), establece que los y las ecuatorianas son racistas y machistas, esto se demuestra en su diario vivir, sin embargo, no son capaces de reconocer estas actitudes o acciones de exclusión y discriminación, lo cual facilita que se ignore la realidad de las mujeres racializadas. (p. 108)

El no reconocimiento de la mujer parte de un proceso prolongado que utiliza herramientas derivadas de las actitudes machistas y misóginas, mismas que hacen que la sociedad considere menos importante su realidad, la cual no necesitaría ser analizada y mucho menos cuestionada, entre las más comunes están: el mansplaining, utilizado para menospreciar los conocimientos de una mujer (Vaccaro, 2021, p. 199); el manterrupting, que encuentra la necesidad de interrumpir las diferentes intervenciones femeninas sin motivos sólidos (Leal, 2019, p. 355); y el gaslighting que invalida las expresiones de las mujeres so pretexto de su mala salud mental (Galán \& Figueroa, 2017, pp. 57-58); lo que permite que en la convivencia diaria se normalice la violencia hacia las ecuatorianas, dado que la mayoría de hombres piensan que tienen la potestad para menospreciarlas, interrumpirlas e invalidarlas. De acuerdo con la segunda interrogante, las consecuencias son directas para las mujeres, y se evidencian en su diario vivir. El abandono del Estado y la sociedad provocan que las mujeres sean sujetas de violencia por condiciones de género. Según los datos de la Fiscalía General del Estado (2020), las mujeres prefieren no denunciar a sus agresores y esto evidencia la falta de apoyo por parte de aparato estatal. Adicional a eso, las mujeres se han sentido invalidadas dentro de los espacios políticos, (ONU MUJERES \& CNE, 2019) establece que la paridad numérica entre hombres y mujeres dentro de un partido no 
garantiza que sean tratados como iguales en los procesos (p. 29). En el campo de la ciencia y la investigación también se refleja esta problemática, (Pessina, 2019) afirma que la igualdad legal no basta para que las mujeres se desenvuelvan bajo las mismas condiciones de los varones, y a su vez los prejuicios patriarcales afectan a sus procesos investigativos (pp. 53 - 54). La esfera deportiva no es la excepción (Ordóñez, 2011) manifiesta que existe un porcentaje mínimo de mujeres deportistas, pues han visto las mismas limitaciones que en otros aspectos sociales (pp. 112-113). Finalmente, que el trabajo doméstico sea asignado socialmente a las mujeres, es un factor que contribuye a su invisibilización, dado que las desvaloriza y las limita en su desarrollo.

\section{Tabla 1}

Breve descripción de las condiciones en las que se desarrollan las mujeres ecuatorianas

\begin{tabular}{|c|c|c|c|}
\hline \multicolumn{4}{|c|}{ MUJERES } \\
\hline & Afroecuatorianas & Indígenas & Lesbianas Trans \\
\hline $\begin{array}{l}\text { Economía y } \\
\text { trabajo. }\end{array}$ & $\begin{array}{l}\text { Una gran mayoría } \\
\text { tiene } \\
\text { informal, } \\
\text { ingresos fijos, a esta } \\
\text { situación se le suma } \\
\text { el poder patriarcal } \\
\text { que controla las } \\
\text { plazas de trabajo. } \\
\text { (COMUNAME \& } \\
\text { FES-ILDIS, 2021) }\end{array}$ & $\begin{array}{l}\text { La exclusión económica de las } \\
\text { indígenas las convierte en } \\
\text { invisibles ante el Estado y } \\
\text { dificulta el denunciar las } \\
\text { violaciones hacia sus derechos } \\
\text { humanos, } \\
\text { vulnerables. } \\
\text { Interamericana de } \\
\text { Humano, } \\
\text { Humdolas } \\
\text { (Comisión } \\
\end{array}$ & $\begin{array}{lrr}\text { No existen } & \text { datos } \\
\text { estadísticos } & \text { que } \\
\text { reflejen a } & \text { nivel } \\
\text { nacional } & & \text { la } \\
\text { situación } & & \\
\text { económica } & \text { y } & \text { de } \\
\text { trabajo, } & & \text { sin } \\
\text { embargo, } & & \text { de } \\
\text { acuerdo con } & \text { los } \\
\text { datos de. (INEC \& } \\
\text { CDT, 2013) } & 49,5 \% \\
\text { de la PEA ocupada } \\
\text { son trabajadoras de } \\
\text { los servicios } \\
\text { vendedores } & \text { de } \\
\text { comercios } & \text { y } \\
\text { mercados. } & \end{array}$ \\
\hline \multirow{2}{*}{ Salud } & \multirow{2}{*}{$\begin{array}{l}\text { Se ha borrado de la } \\
\text { historia los } \\
\text { conocimientos de } \\
\text { medicina tradicional } \\
\text { de las mujeres afro, } \\
\text { sus conocimientos y } \\
\text { roles como } \\
\text { curanderas. (Méndez } \\
\text { D. , 2018) }\end{array}$} & $\begin{array}{l}\text { Falta de personal bilingüe, de } \\
\text { información y y de } \\
\text { infraestructura. (Consejo de } \\
\text { Regulación y Desarrollo de la } \\
\text { Información y Comunicación, } \\
\text { 2017) }\end{array}$ & $\begin{array}{lr}\text { Son excluidas y } \\
\text { omitidas de planes } \\
\text { de prevención y } \\
\text { tratamiento de } \\
\text { enfermedades de } \\
\text { transmisión sexual. } \\
\text { La hetero }\end{array}$ \\
\hline & & $\begin{array}{l}\text { Los derechos sexuales y } \\
\text { reproductivos de las mujeres } \\
\text { indígenas son invisibilizados } \\
\text { por parte del sector público y } \\
\text { privado. (Ortiz, 2009) }\end{array}$ & $\begin{array}{l}\text { sexualización y la } \\
\text { información } \\
\text { limitada genera que } \\
\text { ellas se alejen de } \\
\text { los sistemas de } \\
\text { salud. (Álvarez \& } \\
\text { Cordero, 2017). }\end{array}$ \\
\hline
\end{tabular}




\begin{tabular}{|c|c|c|c|}
\hline Educación & $\begin{array}{l}\text { Del } 14,4 \% \text { de } \\
\text { población } \\
\text { afrodescendiente el } \\
7,9 \% \text { representa a las } \\
\text { mujeres analfabetas. } \\
\text { (INEC, 2010) }\end{array}$ & $\begin{array}{l}1 \text { de cada } 10 \text { niñas indígenas } \\
\text { termina la escuela secundaria } \\
\text { en Latinoamérica. (Comisión } \\
\text { Interamericana de Derechos } \\
\text { Humano, 2017) }\end{array}$ & $\begin{array}{l}\text { No existe una } \\
\text { temática educativa } \\
\text { que hable sobre } \\
\text { vivencias, } \\
\text { necesidades } \\
\text { realidades y } \\
\text { lesbianas y trans. }\end{array}$ \\
\hline \multirow[b]{2}{*}{ Reconocimiento } & $\begin{array}{l}\text { En Ecuador se utiliza } \\
\text { indistintamente a } \\
\text { categorías raciales } \\
\text { como "negro" sin } \\
\text { tomar en cuenta } \\
\text { elementos } \\
\text { conceptuales } \\
\text { metodológicos. } \\
\text { (Antón, 2010) }\end{array}$ & $\begin{array}{l}\text { Los logros conseguidos por el } \\
\text { movimiento indígenas se ven } \\
\text { ocupados con la violencia } \\
\text { sistemática que sufren las } \\
\text { mujeres indígenas. (Lavinas, } \\
2009 \text { ) }\end{array}$ & $\begin{array}{l}\text { No tienen } \\
\text { representación } \\
\text { política, ni social. }\end{array}$ \\
\hline & $\begin{array}{l}231 \text { mil mujeres son } \\
\text { excluidas } \\
\text { Ecuador, situación } \\
\text { que se agrava con } \\
\text { aquellas que se auto } \\
\text { consideran negras, } \\
\text { montubias y mulatas. } \\
\text { (King \& Morales, } \\
2018)\end{array}$ & $\begin{array}{l}\text { En las comunidades indígenas } \\
\text { existen distintas violencias de } \\
\text { género. (Méndez G. , 2009) }\end{array}$ & $\begin{array}{l}\text { Solo existe un } \\
\text { pequeño estudio de } \\
\text { caso por parte de } \\
\text { INEC para tener } \\
\text { datos porcentuales } \\
\text { mínimos, en este } \\
\text { estudio solo se } \\
\text { tomaron en cuenta } \\
\text { a } 2805 \text { personas de } \\
\text { las principales } \\
\text { ciudades del país. }\end{array}$ \\
\hline
\end{tabular}

\section{Fuente: Elaboración propia}

En la Tabla 1 se describe la realidad de las mujeres afroecuatorianas, indígenas, lesbianas y trans que, como consecuencia de ser parte de grupos minoritarios, sufren discriminación y doble invisibilización.

Las consecuencias de la invisibilización también implican el no tomar en cuenta a mujeres neurodivergentes, con discapacidad física, migrantes, rurales, montubias, aquellas que se encuentran en el espectro de lo no binario y demás, dado que el sistema genera barreras de reconocimiento, el no tener en cuenta sus contextos, limita la creación de mejores condiciones de vida para su desarrollo.

Finalmente, respecto a la respuesta de la última interrogante relacionada al aporte de la mujer, es fundamental destacar que, a pesar de no ser reconocidas y de no poseer las mismas ventajas que los hombres, la mujer ecuatoriana ha logrado romper paradigmas de las opresiones sistemáticas y destacar en las distintas esferas de la sociedad, desde el reconocimiento individual o colectivo. En lo económico, ellas son un pilar fundamental, Acosta (2019), afirma que las actividades realizadas las mujeres son representativas en el crecimiento económico del país. (p. 53). En la participación política, Ortiz (2012), establece 
que la lucha de las mujeres les ha permitido conseguir espacios de participación dentro de lo social y político. (p. 85).

Las mujeres han aportado a la ciencia, tecnología, política, economía, educación y lucha social reivindicativa. Además, se debe resaltar la creación de redes de ayuda y espacios seguros que han generado para resistir al sistema que las violenta. (Ver Tabla 2)

\section{Tabla 2}

\section{Aporte de la mujer ecuatoriana}

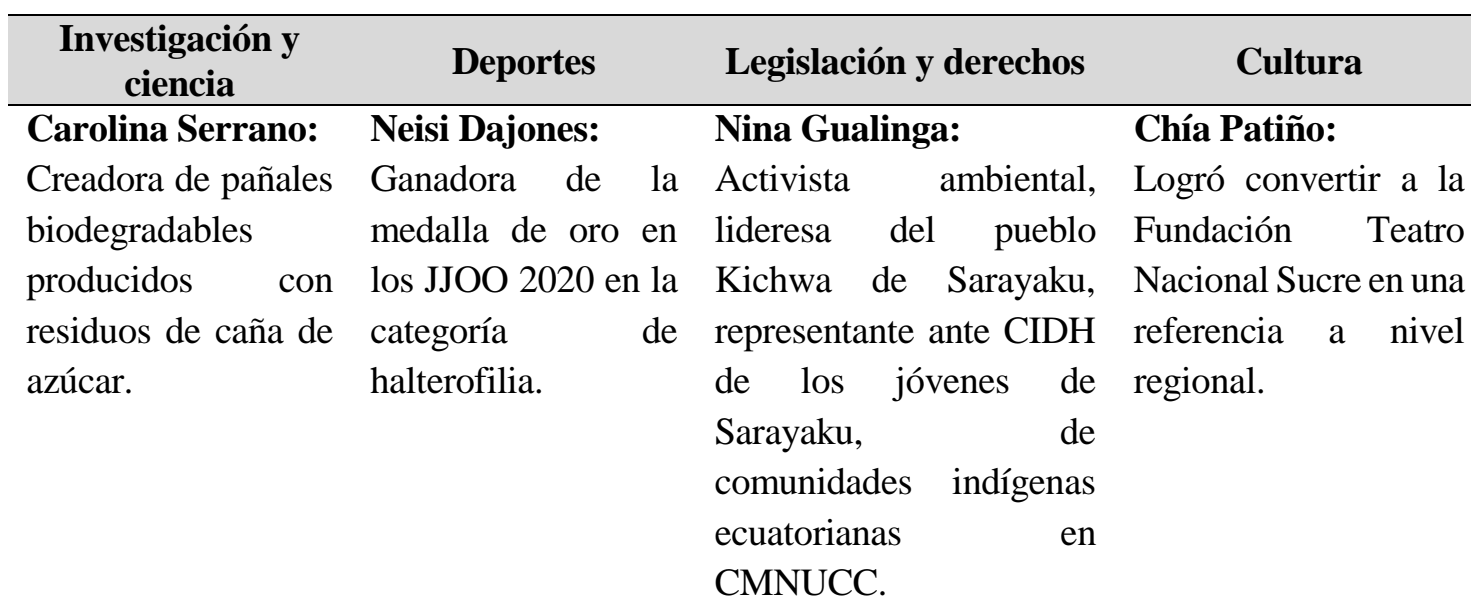

\begin{tabular}{lrlrrrr}
\hline María Cazorla: & Tamara Salazar: & Patricia & Gualinga: & Mónica & Ojeda: \\
Promotora de una & Pesista & Lideresa de Sarayaku & Primera & mujer \\
estación & de & afroecuatoriana, & presidenta de la & ecuatoriana artista en \\
mediciones & & ganó la medalla de & Fundación de Derechos & ganar el premio \\
atmosféricas & y & plata en halterofilia & Humanos del TIAM, Príncipe Claus en \\
pionera en el & en Tokio 2020. & activista en contra de la & 2019. & \\
desarrollo de ISAF, & & explotación petrolera de & \\
científica de la Nasa. & & su territorio y a favor de \\
& & los derechos indígenas y \\
& de la naturaleza.
\end{tabular}

María Endara: Poleth Méndez: Shiram $\quad$ Diana Gabriela Calvache:

Bióloga evolutiva, Ganadora de la Atamaint: pertenece a Su cortometraje En puso en duda la medalla de oro en la comunidad Shuar, espera ganó el premio teoría de la lanzamiento de bala lucha por la mejora de al Mejor Corto de coevolución. en los Juegos las condiciones de vida Ficción de Clasificó e Paralímpicos 2020 de shuar, achuar y Latinoamérica de la identificó nuevas en la categoría F20. mestizos. Primer mujer televisión de Brasil. especies de árboles. shuar que forma parte de Con el cortometraje la academia de la UASB. La mala noche ganó el premio a Mejor película internacional en el NY Latino Film Festival HBO (La Hora, 2020). 


\begin{tabular}{|c|c|c|c|}
\hline \multirow{5}{*}{$\begin{array}{l}\text { María Ulloa: } \\
\text { Publicó la existencia } \\
\text { de } \quad 22 \text { plantas } \\
\text { ecuatorianas nuevas } \\
\text { para la ciencia. }\end{array}$} & Anís Méndez: & \multirow{2}{*}{$\begin{array}{l}\text { Varios colectivos: } \\
\text { Presentación de acción de }\end{array}$} & Paulina Aguirre: \\
\hline & Obtuvo la medalla & & Primera mujer en \\
\hline & de bronce en los & inconstitucionalidad del & obtener un Grammy \\
\hline & juegos paralímpicos & artículo 150 COIP, & Latino en la categoría \\
\hline & $\begin{array}{l}\text { en el lanzamiento de } \\
\text { bala en la categoría } \\
\text { F20. }\end{array}$ & $\begin{array}{l}\text { numeral 2, dando paso al } \\
\text { debate de la legalización } \\
\text { del aborto en casos de } \\
\text { violación. }\end{array}$ & $\begin{array}{l}\text { Mejor Álbum } \\
\text { Cristiano en español. }\end{array}$ \\
\hline Linda & & Johanna & Susana Reyes: \\
\hline $\begin{array}{l}\text { Microbióloga, } \\
\text { proyecto para }\end{array}$ & $\begin{array}{l}\text { Logró obtener la } \\
\text { medalla de bronce }\end{array}$ & $\begin{array}{lr}\text { para } & \text { el } \\
\text { de } & \text { Ley }\end{array}$ & $\begin{array}{lr}\text { Su aporte más } \\
\text { destacado es la }\end{array}$ \\
\hline incorporar & en los Paralímpicos & Orgánica de Salud e & "Danza Butoh" de los \\
\hline propiedades de la & de Tokio en la & Higiene Menstrual. & Andes y la creación \\
\hline $\begin{array}{l}\text { carne de origen } \\
\text { animal a la carne de } \\
\text { origen vegetal. }\end{array}$ & $\begin{array}{l}\text { categoría T46 de } \\
\text { largo salto. }\end{array}$ & & $\begin{array}{l}\text { de la Casa de la } \\
\text { Danza. }\end{array}$ \\
\hline
\end{tabular}

\section{Fuente: Elaboración propia}

La Tabla 2 recoge los aspectos más importantes del aporte de la mujer ecuatoriana, muestra su protagonismo en el campo de la investigación, ciencia, deportes, legislación, derechos y cultura, a pesar de las grandes dificultades que enfrentan y limitaciones impuestas por los múltiples sistemas de opresión.

\section{CONSIDERACIONES FINALES}

- La invisibilización de la mujer ecuatoriana no es un proceso espontáneo, es una problemática social que se ha forjado históricamente, se fundamenta en el no reconocimiento y la marginalización de sus vivencias y necesidades, siendo vulnerables a todas las formas de violencia que se desprenden del carácter de clase e inequitativo de la sociedad ecuatoriana.

- La causa directa de esta invisibilización se encuentra en el androcentrismo, que pone al hombre como centro de la humanidad y ha excluido a las mujeres de todas las narrativas socioculturales, lo que genera el surgimiento del patriarcado, sistema que se asocia fácilmente con el capitalismo y junto a este, desarrollan las condiciones necesarias para violentar a la mayoría de las mujeres; sin embargo, las causas de la invisibilización e inequidades se entretejen entre sí.

- Las consecuencias de la invisibilización de la mujer ecuatoriana se resumen en sus condiciones de vida y desarrollo. La pobreza, educación inconclusa, analfabetismo, violencia y el restringido acceso a los derechos básicos son parte de las afectaciones que viven las mujeres ecuatorianas, estas pueden ser más graves en algunos casos. 
Además, como una consecuencia generalizada son las largas jornadas de trabajo que pueden llegar a tener, provocando limitaciones en su desarrollo. El ignorar esta invisibilización ayuda a replicar situaciones de violencia y vulnerabilidad.

- Los aportes de las mujeres ecuatorianas a pesar de las barreras impuestas tienen un significado importante para la sociedad ecuatoriana. Sus contribuciones se evidencian en la economía, política, ciencia, investigación, cultura y educación, y particularmente, se debe destacar su protagonismo en lucha reivindicativa por conseguir autonomía y equidad.

- Es importante luchar para que mujeres y hombres tengan igualdad de oportunidades y de trato, sin discriminación y exclusión, en ejercicio pleno de sus derechos establecidos en la Constitución y demás instrumentos de derechos humanos; por un país intercultural y plurinacional donde se promueva el diálogo, el respeto a la diversidad, la participación y la equidad de género.

\section{LISTA DE REFERENCIAS}

Acosta, L. (2019). La evaluación del aporte de las mujeres en la economía ecuatoriana, en el período 2013 al 2017. Guayaquil: Universidad de Guayaquil. Recuperado de http://repositorio.ug.edu.ec/bitstream/redug/49308/1/TESIS-

LIZ\%20GENOVA\%20ACOSTA\%20ACOSTA.pdf

Álvarez, S., \& Cordero, M. (2017). Diagnóstico participativo sobre la situación de los derechos humanos de la población LGBTI. Cuenca: Sendas, Hivos, UE. Recuperado

de http://iessdeh.org/usuario/ftp/Diagnostico_DDHH_LGBTI_Ecuador_Publicacio n.pdf

Amoros, C. (1992). Notas para una teoría nominalista del patriarcado. Asparkia: Investigación feminista (1), 41-58. Recuperado de https://www.erevistes.uji.es/index.php/asparkia/article/view/412/331

Antón, J. (2010). La experiencia afrodescendiente y la visibilidad estadística en el Ecuador. Santiago de Chile: Organización de las Naciones Unidas. Recuperado de

https://www.cepal.org/sites/default/files/publication/files/39978/LCW368_es.pdf

Arruza, C. (2014). Reflexiones degeneradas: Patriarcado y capitalismo, 2014. Disponible en: Worpress. Acceso en: octubre 2018. Recuperado de 
https://marxismocritico.files.wordpress.com/2016/03/reflexiones-dege-neradaspatriarcado-y-capitalismo.pdf

Coello, M. (2016). Influencia de los grupos de presión de mujeres afroecuatorianas en la discriminación racial y de género (2006-2013). Quito: PUCE. Recuperado de http://8.242.217.84:8080/xmlui/bitstream/handle/123456789/2472/INFLUENCI A\%20DE\%20LOS\%20GRUPOS\%20DE\%20PRESI\%c3\%93N\%20DE\%20MUJ ERES\%20AFROECUATORIANAS\%20EN\%20LA\%20DISCRIMINACI\%c3\% 93N\%20RACIAL\%20Y\%20DE\%20.pdf?sequence=1\&isAllowed=y

Comisión Interamericana de Derechos Humano. (2017). Las mujeres indígenas y sus derechos humanos en las Américas. Dinamarca: Organización de Estados Americanos. http://www.oas.org/es/cidh/informes/pdfs/MujeresIndigenas.pdf

COMUNAME \& FES-ILDIS. (2021). Diagnóstico de la situación socioeconómica de las mujeres afroecuatorianas en el territorio ancestral de Imbabura y Carchi. Quito: Friedrich-Ebert-Stiftung Ecuador FES-ILDIS. Obtenido de http://library.fes.de/pdf-files/bueros/quito/17548.pdf

Consejo de Regulación y Desarrollo de la Información y Comunicación. (2017). Representaciones y estereotipos hacia las mujeres en los medios de comunicación. Quito: Consejo de Regulación y Desarrollo de la Información y Comunicación. Recuperado de https://biblio.flacsoandes.edu.ec/libros/digital/56758.pdf

Fiscalía General del Estado y Escuela Politécnica Nacional. (2020). Análisis de la violencia de género. Recuperado de Fiscalía General del Estado: https://www.fiscalia.gob.ec/estadisticas-fge/

Frega, M. (2019). Que el capitalismo y el patriarcado caigan juntos. Apuntes sobre las potencialidades, límites y desafíos de los feminismos en la experiencia argentina reciente. Theomai (39), 21-38. Recuperado de https://www.redalyc.org/jatsRepo/124/12466126003/12466126003.pdf

Galán, J., \& Figueroa, M. (2017). Gaslighting: La invisible violencia psicológica. UARICHA. Revista de Psicología, 14(32), 53-60. Recuperado de http://www.revistauaricha.umich.mx/ojs_uaricha/index.php/urp/article/view/151 $/ 137$ 
Hernández, I. (2020). La invisibilización como metáfora: Una categoría de análisis para identificar el proceso de invisibilización en problemáticas sociales. Trama. Revista de Ciencias Sociales y humanidades, 9(1), 100 - 131. Recuperado de Trama. Revista de Ciencias Sociales y humanidades: https://revistas.tec.ac.cr/index.php/trama/article/view/5271/4938

Honnet, A. (2011). Invisibilidad. Sobre la epistemología moral del "reconocimiento". In A. Honnet, La sociedad del desprecio (pp. 165-181). Madrid: Trotta. Recuperado de https://www.uv.es/fjhernan/py/Honneth2.pdf

INEC \& CDT. (2013). Estudio de caso sobre condiciones de vida, inclusión social cumplimiento de derechos humanos de la población LGBTI en el Ecuador. Quito: INEC \& CDT. Recuperado de https://www.ecuadorencifras.gob.ec/documentos/webinec/Estadisticas_Sociales/LGBTI/Analisis_situacion_LGBTI.pdf

INEC. (2010). Censo de Población y Vivienda. Quito: INEC.

INEC. (2019, noviembre). ENCUESTA NACIONAL SOBRE RELACIONES FAMILIARES Y VIOLENCIA DE GÉNERO CONTRA LAS MUJERES. Recuperado de https://www.ecuadorencifras.gob.ec/documentos/webinec/Estadisticas_Sociales/Violencia_de_genero_2019/Principales\%20resultados \%20ENVIGMU\%202019.pdf

INEC. (2020). Cuentas Satélite del Trabajo No Remunerado de los Hogares 2016 -2017. Quito: INEC. Recuperado de https://www.ecuadorencifras.gob.ec/documentos/webinec/Estadisticas_Economicas/Cuentas_Satelite/Cuenca_satelite_trab_no_remun _2017/2_Presentacion_resultados_CSTNRH.pdf

King, K., \& Morales, J. (2018). ¿Quiénes son las mujeres faltantes en el Ecuador? Una aproximación cuantitativa. Analítika. Revista de análisis estadístico., 16(2), 4567. Recuperado de https://www.ecuadorencifras.gob.ec/documentos/webinec/Revistas/Analitika/Anexos_pdf/Analit_16/2a.pdf

Lavinas, M. (2009). La violencia como factor de exclusión política: mujeres indígenas en Chimborazo. In A. Pequeño, Participación y políticas de mujeres indígenas en contextos latinoamericanos recientes (pp. 125-143). Quito: FLACSO; Ministerio 
Invisibilización de la mujer ecuatoriana...

de

Cultura.

Recuperado

de

https://biblio.flacsoandes.edu.ec/catalog/resGet.php?resId=41461

Leal, A. (2019). Reseña de: Rebecca Solnit. Los hombres me explican cosas. Madrid, Capitán Swing, 2016. 143 pp. Título original: Men explain things to me, 2015. Traducción de Paula Martín Ponz. ISBN: 978-84-945481-4-7. Feminismo/s (34), 335-360.

Recuperado

de: https://rua.ua.es/dspace/bitstream/10045/100419/1/Feminismos_34_17.pdf

Méndez, D. (2018). Participación de organizaciones sociales de mujeres afroecuatorianas en el reconocimiento del pueblo afroecuatoriano establecido en la constitución del Ecuador en el año 2008. Quito: FLACSO. Recuperado de http://8.242.217.84:8080/xmlui/bitstream/handle/123456789/3555/TFLACSO2018DIMP.pdf? sequence $=2 \&$ isAllowed $=\mathrm{y}$

Méndez, G. (2009). Miradas de género de las mujeres indígenas en Ecuador, Colombia y México. En A. Pequeño, Participación y políticas de mujeres indígenas en contextos latinoamericanos reciente (págs. 53-71). Quito: FLACSO; Ministerio de Cultura.

ONU MUJERES \& CNE. (2019, octubre). Estudio Violencia Política contras las mujeres en el Ecuador. Recuperado de ONU: https://ecuador.un.org/sites/default/files/202002/violencia\%20politica\%20baja.p df

Ordóñez, A. (2011). Género y deporte en la sociedad actual. Polémika - Publicación Semestral de la Universidad San Francisco de Quito, 3(7), 106-113. Recuperado de https://revistas.usfq.edu.ec/index.php/polemika/article/view/404/381

Ortiz, J. (2009). Derechos sexuales y reproductivos en los pueblos originarios del Ecuador. Cuenca: Universidad de Alicante. Recuperado de https://rua.ua.es/dspace/bitstream/10045/16050/1/tesis_JoseOrtiz.pdf

Pessina, M. (2019). Ecuador: Investigadoras en Ciencia y Tecnología atrapadas entre la invisibilización y el androcentrismo. In OEI, CIESPAL, \& R. \&. SENECYT, Impacto de las Mujeres en la Ciencia Efecto del género en el desarrollo y la práctica científica (pp. 29-55). Quito: Organización de Estados Iberoamericanos. Recuperado de https://ciespal.org/wp-content/uploads/2019/06/Mujeres-en-lacienciaDigital-VFJUL19.pdf 
Radcliffe, S. (2014). El género y la etnicidad como barreras para el desarrollo: Mujeres indígenas, acceso a recursos en Ecuador en perspectiva latinoamericana. Eutopía: Revista de Desarrollo Económico Territorial, 1(5), 11-34. Recuperado de https://dialnet.unirioja.es/descarga/articulo/5040152.pdf

Vaccaro, V. (2021). Women make film. A new road movie through cinema de Mark Cousins. FILMHISTORIA Online, 31(1), 197-201. Recuperado de https://revistes.ub.edu/index.php/filmhistoria/article/download/35541/34817 